\title{
El medio audiovisual y el imaginario social de las personas con discapacidad auditiva
}

\section{The audiovisual media and the social imaginary of people with hearing disabilities}

DOI: https://doi.org/10.29166/tyc.v1i21.2389

\section{Jorge Delgado Rocha}

Estudió en la Universidad Técnica Nacional de Vinnytsia (Ucrania) donde se graduó, en 2013, como Ingeniero en Administración de Empresas especializado en Relaciones Internacionales. En 2015 obtuvo su maestría en la misma rama. Es profesor de la Facultad de Comunicación Social en la Universidad Central del Ecuador y traductor independiente de ruso. Sus principales temas de investigación incluyen la motivación laboral, la investigación sobre cultura organizacional, manejo de gobierno electrónico y gestión de marcas.

\section{Josselyn Fuentes González}

En 2020 terminó su formación universitaria en la Facultad de Comunicación Social de la Universidad Central del Ecuador, con énfasis en Periodismo. Trabajó como docente en el Instituto de Audición y Lenguaje Enriqueta Santillán. Ha realizado talleres de formación cinematográfica en el Laboratorio Comunitario Audiovisual Ojo Semilla con el programa de becas auspiciado por la Fundación El Churo.

Correo: jgfuentes@uce.edu.ec

\section{Resumen}

El presente artículo se enfoca en el uso de los medios audiovisuales como herramientas para representar el imaginario social de las personas con discapacidad auditiva. Para ello, se usará la cartografía social como método cualitativo para determinar las percepciones de este grupo acerca de la sociedad que lo rodea. Estas percepciones se representarán en forma de video para mostrar la capacidad de los medios audiovisuales de conectarse y entender diferentes realidades sociales.

Palabras clave: cartografía, medio audiovisual, imaginario social, discapacidad auditiva, lenguaje.

\section{Abstract}

This article focuses on the audiovisual media usage as tools to represent the social imaginary about people with hearing disabilities. To this, social cartography will be used as a qualitative method to determine the perceptions that this group of people have about the society in which they operate. These perceptions will be represented in video to demonstrate the capacity of audiovisual media to connect and understand different social realities.

Keywords: mapping, audiovisual media, social imaginary, hearing impairment, language. 


\section{El lenguaje de señas a través del audiovisual}

En la actualidad los avances tecnológicos nos permiten alcanzar una vida más sencilla. $\mathrm{Al}$ mismo tiempo podemos percibir que existen sectores que se ven imposibilitados de ejercer una interacción con el resto. A este fenómeno, algunas corrientes de pensamiento lo suelen llamar "estigma”.

Las personas con discapacidad auditiva tienen que vivir en una especie de burbuja cuasi impenetrable en la que resulta difícil saber de qué manera se comunican. Muchas veces no se le otorga suficiente relevancia a este tema y esto puede generar un grave problema para las personas con discapacidad auditiva: su invisibilidad. El objetivo primordial de esta investigación es demostrar que, mediante el uso de herramientas audiovisuales, se puede crear mensajes con el lenguaje de señas que nos permitirán entender, visibilizar y acercarnos a la realidad de este grupo de personas.

Según Tyler (2018), basado en las reflexiones de Erving Goffman respecto del estigma, éste se manifiesta en la antigüedad griega, cuando se crea el término para referirse a signos corporales con los que se pretendía exhibir algo malo y poco habitual en el estatus moral de quien lo presentaba. Actualmente, se usa la palabra de manera amplia para referirse al mal en sí mismo y no a sus manifestaciones corporales.

Es en las interacciones sociales de quienes no encajan con el prototipo de "normales" donde se producen los llamados "estigmas". En este caso, respecto de los niños y niñas que presentan discapacidad auditiva. Ellos son objeto de conductas discriminatorias como, por ejemplo: críticas a través del lenguaje. Son estas las que, poco a poco, influyen en su aislamiento social. Esto se manifiesta al momento de tratar de incorporarse a la sociedad que los rodea, que los hace sentir menos, restando su posibilidad de asumir un papel dentro de esta.

Álvarez (2015) establece una relación entre las personas con discapacidad y los mecanismos de gestión y resolución de conflictos. Esto supone mostrar dos fenómenos que se han visibilizado recientemente, aunque su existencia es antigua. La primera es un trabajo que aporte al cambio social y jurídico para el ejercicio pleno de los derechos y del estatus de ciudadanía de las personas con discapacidad. La segunda es la promoción de los sistemas alternativos de prevención, gestión y resolución de conflictos para garantizar el pleno ejercicio de la igualdad y la inclusión de este sector de la población. “...La preocupación principal de esta perspectiva es que la discapacidad no emerge de la incapacidad intrínseca sino preponderantemente del modo en que las necesidades de las personas son satisfechas". (Álvarez, 2015, pág. 31).

Este análisis plantea una alternativa comunicacional para las personas con discapacidad auditiva: el audiovisual como principal insumo para visibilizar los problemas y lograr una comunicación eficaz de las personas en esta situación con la sociedad en general.

La comunicación mediante el lenguaje de señas usa un núcleo similar al lenguaje oral: la palabra. La designación de una palabra adecuada para un objeto es fundamental para materializar lo real y comprender el entorno que nos rodea. La generalización de estas palabras y su significado permite la interacción y com- 
prensión de las personas, que expresan sus pensamientos mediante la articulación de estas palabras. En el caso de las personas con discapacidad auditiva, al no poder emitir palabras mediante el lenguaje convencional, usan el lenguaje de señas. (ver Tabla 1)

Al tratarse de un lenguaje netamente visual, el significante se limita a la seña realizada con las manos o con la expresividad del rostro o del cuerpo. En la mente del receptor se maquinará la imagen de la palabra, más no su escritura gramatical. El significado será el mismo y no tendría cabida la dimensión física ya que no se emite ninguna clase de sonido.

En el caso de las personas con discapacidad auditiva, el mundo de lo real se representa mediante las señas que reflejan la similitud con los objetos. Esto implica la creación de un lenguaje exclusivo para su comunicación.

Dentro del acto comunicativo podemos encontrar la comunicación verbal y la no verbal. Según Cestero Mancera (2006) las expresiones no verbales aluden a los sistemas de signos no lingüísticos que se utilizan para comunicar. Se trata de un concepto amplio que incluye los hábitos y las costumbres culturales.

La comunicación no verbal está influenciada por los hábitos y las costumbres culturales del hablante $\mathrm{y}$ tiene relación directa con el entorno que lo rodea. Esta arista de la comunicación tiene como objetivo, según Cestero Mancera (2006), el estudio de los signos y actos de comunicación producidos a partir de signos. Para este autor, el sistema quinésico está formado por movimientos, posturas corporales que comunican o matizan el significado de los enunciados verbales. Se incluyen dentro de estos movimientos a la mirada y el contacto corporal.

Dentro de este sistema podemos encontrar tres categorías: a) Los gestos o movimientos faciales y corporales, entre los cuales se puede distinguir dos tipos de

Tabla 1. Proceso de construcción de la lengua de señas

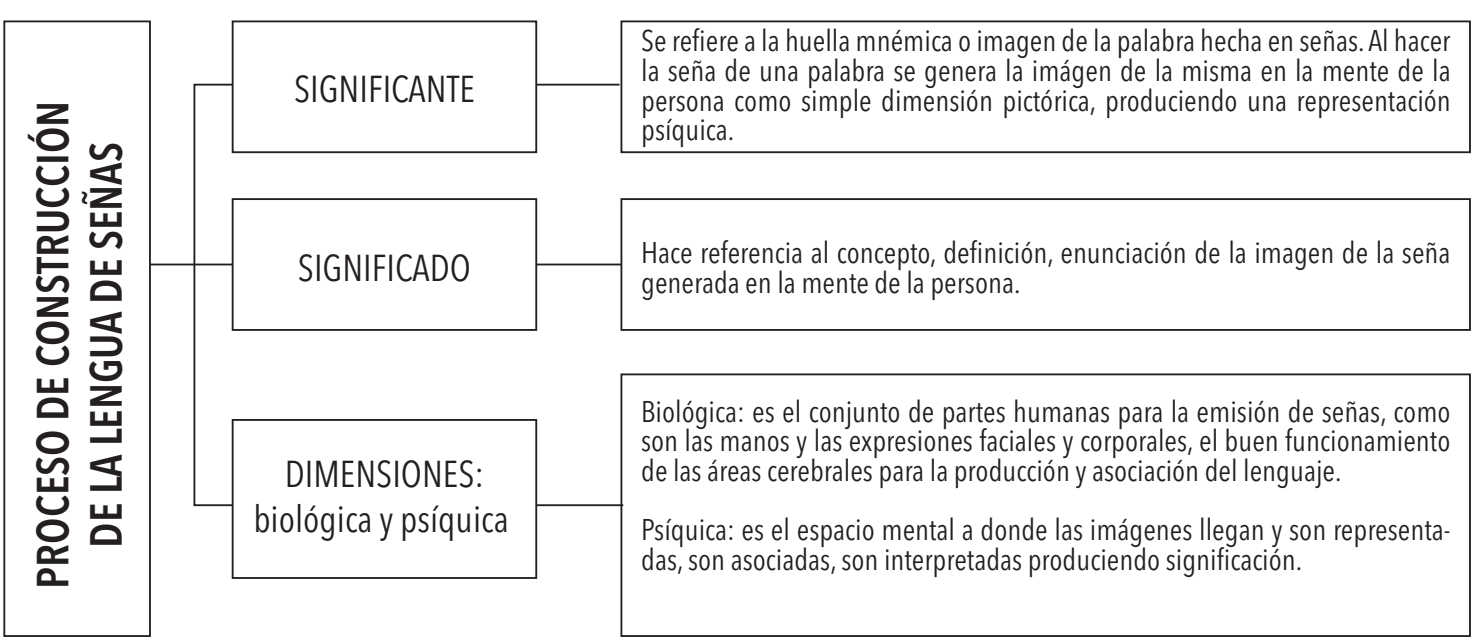

Fuente: Elaboración propia. 
gestos básicos que están interrelacionados. Mancera sostiene que es difícil encontrar gestos simples producidos con un solo órgano y que lo usual es poner en funcionamiento varios órganos a la vez; b) Las maneras o formas convencionales de realizar acciones, movimientos, gestos y posturas; c) Las posturas son las posiciones estáticas que adopta o puede adoptar el cuerpo humano y que comunican, activa o pasivamente.

El sistema proxémico consiste en la distribución que hace el ser humano del espacio natural. El comportamiento de las personas respecto del espacio se relaciona con el orden y desorden en la colocación de objetos, el mantenimiento de filas, el respeto de espacios prohibidos o privados, etc. (Mancera 2006)

\section{Metodología, percepciones y representacio- nes en video}

El Instituto Fiscal de Audición y Lenguaje Enriqueta Santillán, ubicado en el sector de la Mena 2, en el sur de Quito, es la primera institución de Educación Especial de niñas, niños y jóvenes con discapacidad auditiva en el Ecuador. Brinda el servicio de educación desde 1942 y cuenta con el Nivel Inicial, Educación Básica, Bachillerato Técnico y Bachillerato General Unificado. En la actualidad acoge a 100 estudiantes de diferentes edades, con 22 educadores expertos en educación especial y lenguaje de señas. Entre ellos se encuentran cuatro profesores con discapacidad auditiva. En base a la investigación realizada se pudo evidenciar que la mayor parte de los estudiantes de esta institución forman parte del quintil 1 y 2, la población más vulnerable económicamente.

\section{Figura 1. Escudo del Instituto Fiscal} de Audición y Lenguaje Enriqueta Santillán

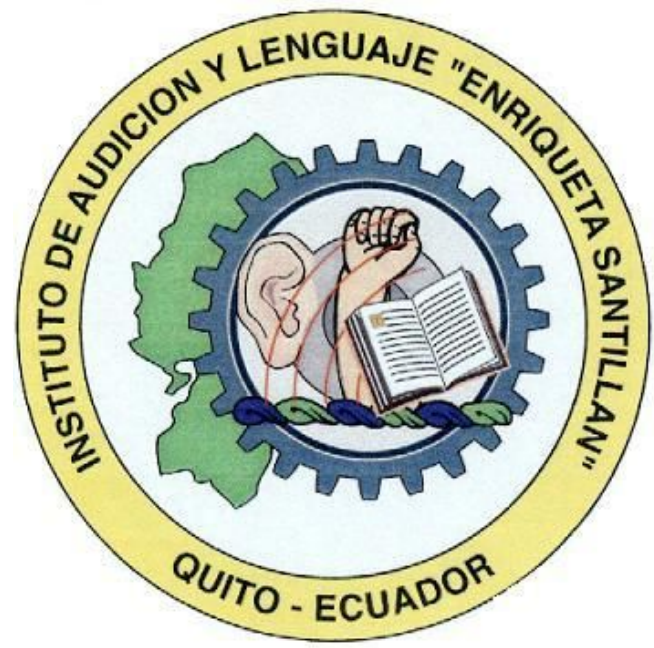

Fuente:Página oficial del Instituto de Audición y Lenguaje "Enriqueta Santillán".

Para visualizar cuáles son las diferentes ideas, percepciones y opiniones que tienen los estudiantes del instituto se utilizará como método de investigación la Cartografía Social. Este procedimiento establece un clima de confianza entre las personas; facilita la manifestación de diversas formas de pensar y de concebir su entorno. Para llevar a cabo esta técnica de investigación participó una moderadora, quien conoce el lenguaje de señas. Esta técnica permite ofrecer a los jóvenes la oportunidad de plasmar sus ideas mediante dibujos e imágenes en mapas.

La cartografía social radica en que también es una opción metodológica para aproximarse a las comprensiones sobre el territorio que un conjunto de seres humanos posee de él. De esta manera, el levantamiento del mapa (cartografía) no se circunscribe a la delimitación de los espacios físicos, sino que se ancla a las diversas 
tensiones que emergen de las relaciones socioculturales de quienes habitan el territorio en cuestión. Importa en esta estrategia el que los diferentes actores lleguen a acuerdos sobre las interpretaciones que emergen de las problemáticas socioculturales que se evidencian en el territorio, de forma tal que, como colectivo, puedan tomar decisiones de transformación de sus prácticas. (Barragán, 2016, p.250)

Esta técnica nos permitirá dimensionar el estado social de las personas sordas. Esto facilitará la búsqueda de una herramienta para satisfacer las necesidades que resulten del análisis. El análisis e interpretación de resultados se realizará mediante cuadros que contengan las acciones de los participantes. Esto con el fin de encontrar similitudes en sus acciones, gestos y actitudes respecto de lo que plasman en el mapa.

El siguiente paso será plasmar esos resultados en un producto audiovisual creado por los propios estudiantes del Instituto de Audición y Lenguaje Enriqueta Santillán. Para ello serán necesarios talleres de capacitación en la producción de piezas audiovisuales y uso de equipo de grabación.

Los talleres se dividirán en 4 fases con un tallerista para cada una:

1. Acercamiento y explicación.

2. Explicaciones técnicas de las cámaras.

3. Explicación de construcción de contenido.

4. Práctica de los estudiantes.

Los participantes del grupo focal realizarán un producto audiovisual con los conocimientos impartidos en los talleres. Definirán su temática y contenido con la guía constante de los talleristas. Se decidió que sean los mismos estudiantes quienes realicen su video de carácter social, ya que de esta manera mantendremos el mensaje intacto, tal y como lo percibieron las personas con discapacidad auditiva.

Se espera que el desarrollo de los productos audiovisuales con los estudiantes brinde una opción real que facilite la interacción de los estudiantes con la sociedad que los rodea. Tomando en cuenta lo que Barrio expresa: “...se presenta el modelo metodológico alcanzado para la producción y desarrollo de contenidos audiovisuales y multimedia para MOOC, tras un año de interacciones sucesivas basadas en ciclos de planificación, acción, observación y reflexión”. (Barrio, 2017, p.185)

\section{Resultados e interpretación}

La cartografía social que se realizó durante enero de 2017 se propuso evidenciar las percepciones de los participantes respecto del rol que cumplen dentro de la sociedad. Para el análisis de la información, se realizó una codificación simple, de la siguiente manera:

- A: respecto de las actitudes positivas de los participantes con relación a las indicaciones o a las actividades que realizaban.

- B: respecto de las especificaciones y características del grupo de trabajo.

- C: respecto de las formas de discriminación que se manifestaban en los mapas.

- D: respecto de la explicación e interpretación que los participantes hacían acerca del producto realizado. 
Textos y contextos $\mathrm{N}^{\mathrm{O}} 21$

\section{Tabla 2. Matriz de datos}

\begin{tabular}{|c|c|c|c|}
\hline PREGUNTA CLAVE & ACCIONES & INDICADOR & CÓDIGO \\
\hline \multirow{4}{*}{$\begin{array}{l}\text { ¿Siempre mantienen esta } \\
\text { actitud? ¿Cómo logran mantener } \\
\text { una actitud positiva a pesar de las } \\
\text { circunstancias? }\end{array}$} & A. 1. Todos saludaban con señas y con amabilidad. & \multirow{4}{*}{$\begin{array}{l}\text { Actitud } \\
\text { positiva }\end{array}$} & \multirow{4}{*}{ A } \\
\hline & $\begin{array}{l}\text { A2. Se observaba un ambiente de tranquilidad y } \\
\text { alegría. }\end{array}$ & & \\
\hline & A3. Mostraron una actitud positiva. & & \\
\hline & A4. Se notó una gran tranquilidad y comodidad. & & \\
\hline $\begin{array}{l}\text { ¿Es equitativo el grupo de trabajo } \\
\text { que se escogió? }\end{array}$ & $\begin{array}{l}\text { B1. El equipo de trabajo estaba conformado por seis } \\
\text { estudiantes: cinco hombres y una mujer. }\end{array}$ & $\begin{array}{l}\text { Especificación } \\
\text { del grupo }\end{array}$ & $B$ \\
\hline \multirow{5}{*}{$\begin{array}{l}\text { ¿La discriminación empieza en } \\
\text { casa? ¿Reciben el apoyo } \\
\text { necesario de su familia? ¿De } \\
\text { quién deberían recibir apoyo } \\
\text { para sobrellevar su discapacidad? } \\
\text { ¿Qué han hecho las autoridades } \\
\text { al respecto? }\end{array}$} & C1. Como viven la discriminación en su vida cotidiana. & \multirow{5}{*}{$\begin{array}{c}\text { Formas de } \\
\text { discriminación }\end{array}$} & \multirow{5}{*}{ C } \\
\hline & $\begin{array}{l}\text { C2. La discriminación con el pasar del tiempo ha sido } \\
\text { menor en su familia. }\end{array}$ & & \\
\hline & $\begin{array}{l}\text { C3. Aun sienten discriminación cuando las personas } \\
\text { oyentes no les toman en cuenta. }\end{array}$ & & \\
\hline & $\begin{array}{l}\text { C4. No pueden alcanzar estudios superiores, por lo cual } \\
\text { no pueden desarrollarse profesionalmente. }\end{array}$ & & \\
\hline & $\begin{array}{l}\text { C5. Las autoridades no tienen paciencia con ellos, } \\
\text { incluso sienten que se burlan cuando quieren } \\
\text { preguntarles algo u obtener algún tipo de información. }\end{array}$ & & \\
\hline \multirow{3}{*}{$\begin{array}{l}\text { ¿Por qué la sociedad no maneja } \\
\text { el idioma de señas? } \\
\text { ¿Es discriminación no } \\
\text { preocuparse por aprender este } \\
\text { idioma? }\end{array}$} & $\begin{array}{l}\text { D1. Con la ayuda de una intérprete nos hacían conocer } \\
\text { por qué escogían esos gráficos y qué significaba para } \\
\text { ellos. }\end{array}$ & \multirow{3}{*}{ Interpretación } & \multirow{3}{*}{ D } \\
\hline & $\begin{array}{l}\text { D2. Poco a poco el cartel se llenaba de gráficos y, para } \\
\text { facilitar la compresión, escribían en la parte inferior lo } \\
\text { que representaba. }\end{array}$ & & \\
\hline & $\begin{array}{l}\text { D3. En las fiestas familiares o de cualquier ámbito no } \\
\text { pueden comunicarse, mucho menos interactuar con } \\
\text { sus familiares ya que la mayoría no conoce el lenguaje } \\
\text { de señas. }\end{array}$ & & \\
\hline
\end{tabular}

Fuente:Elaboración propia 
Se puede observar que las percepciones que tienen los estudiantes del instituto sobre la realidad social en que viven es una sensación de discriminación social y un sentimiento de alejamiento respecto de la sociedad. La mayoría de respuestas en el Código "C" expone las formas de discriminación que viven a diario, tanto en su entorno familiar como en la sociedad en sí.

Todos estos episodios de discriminación radican en la ineficaz interacción comunicativa con las personas del entorno en el que se desarrollan. Esto, a pesar de su deseo por aprender e incorporarse a actividades grupales. En las preguntas del Código "A" los participantes presentaron una actitud proactiva hacia las indicaciones, trabajaron de manera ordenada y evidenciaron signos de compañerismo al trabajar en grupo.

Los estudiantes utilizaron recortes y gráficos para plasmar sus ideas, los pegaron en una cartulina grande y los etiquetaron con marcador. Después de culminar la tarea, cada grupo de trabajo expuso el contenido de esta, lo que signi-

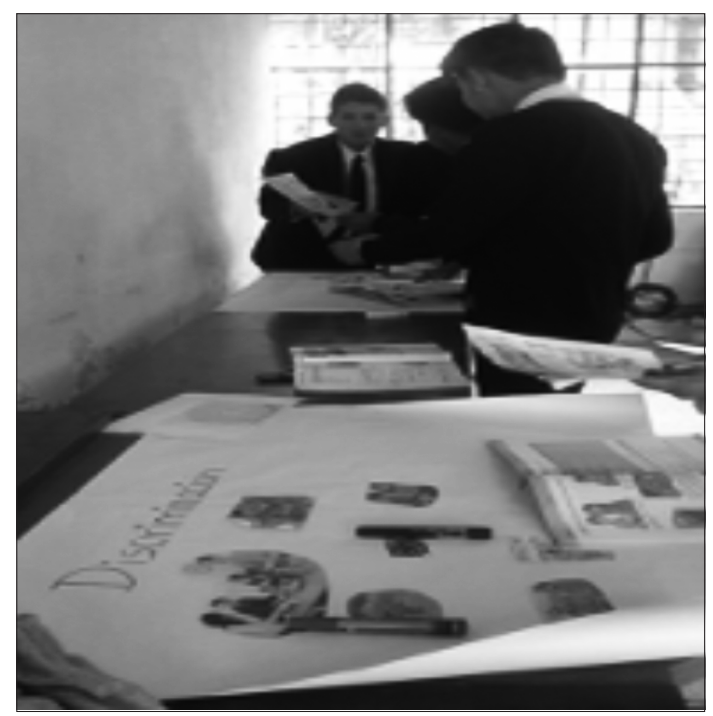

Figura 2. Participación de los estudiantes en la cartografía social fica y representa cada gráfico, respondiendo así a las preguntas de cada código.

El código "B" nos permite observar las especificaciones del grupo y determinar que es un equipo conformado equitativamente. Finalmente se pudo identificar que los espacios de interacción también son relevantes para construir una sensación de discriminación dentro de los estudiantes; Las respuestas del Código " $D$ " se centran en las exposiciones de los mapas y podemos ver los espacios en los que ellos se desenvuelven como: hogar, colegio, espacios públicos, donde expresan sensaciones de incomodidad o molestia.

Los talleres se realizaron los días 14, 15,16 y 17 de febrero de 2017 en jornadas de cuatro horas. En ellos recibieron capacitación sobre la producción en medios audiovisuales. Al finalizar, los estudiantes pusieron en práctica lo aprendido en las jornadas anteriores, tomaron el mando en las grabaciones, organizaron y delegaron responsabilidades entre todo el grupo.

El producto audiovisual conseguido se llama "Un mundo sin sonidos" y tiene una duración de 2 minutos y 32 segundos. Fue construido en relación con las diferencias sociales reflejadas en los resultados de la cartografía social, tomando las más relevantes para los participantes.

Adicionalmente, el feedback de los estudiantes los mostró entusiasmados en aprender esta nueva herramienta que les permite expresar sus pensamientos y sentimientos hacia la sociedad. Juan Holguín expresó: "es bueno que nos tomen en cuenta para este tipo de proyectos porque casi todo el mundo nos ignora y nosotros tenemos mucho que decir. Además, gracias a los videos que podemos hacer, nadie tiene que darnos diciendo [sic] lo que en realidad queremos expresar" (Holguín, 2017). 


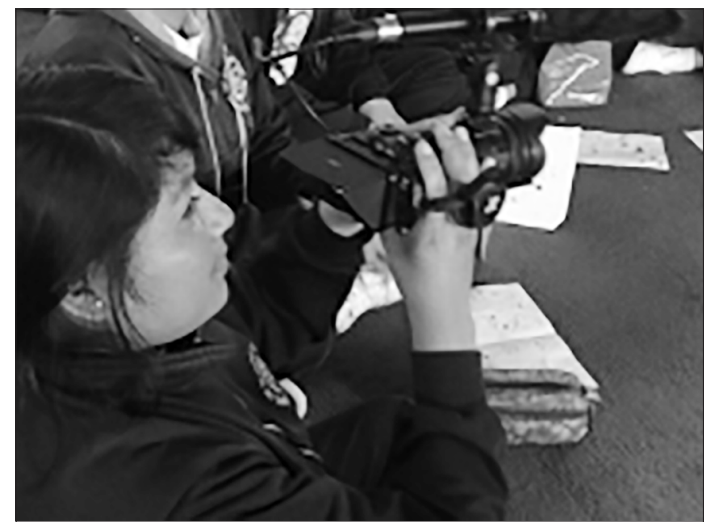

Figura 3. Participantes del taller audiovisual en las grabaciones para el producto final.

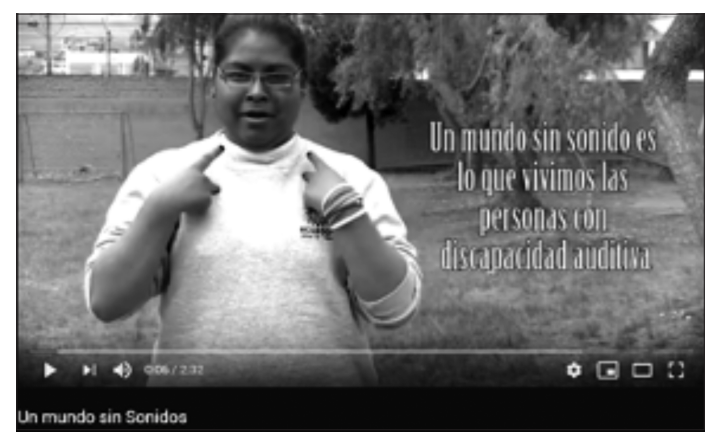

Figura 4. Fotograma del video Un mundo sin sonidos.

Fuente: Página de YouTube de El Churo Comunicación

En los comentarios de los participantes podemos apreciar la principal necesidad que tienen de comunicarse con las personas oyentes de su entorno. Al fallar esta interacción, se producen pensamientos negativos respecto de su condición de discapacidad. De igual manera, se producen sentimientos de soledad y exclusión al ser ignorados. Los talleres cumplieron la función de crear una alternativa comunicativa entre las personas con discapacidad auditiva con la sociedad oyente. Al ser una herramienta amigable para ellos, sintieron seguridad y confort al realizar el producto audiovisual final.

\section{Conclusiones}

Mediante la investigación realizada se ratifica que las personas con discapacidad auditiva, al no mantener una interacción con su entorno de la misma manera que lo hace una persona oyente, perciben la realidad de manera diferente y crean nuevos significados mediante el lenguaje que usan para comunicarse. Esto construye un imaginario exclusivo de las personas con este tipo de discapacidad.

Mediante el análisis de la investigación se pudo observar que el problema de las personas con discapacidad auditiva es mantener una comunicación eficaz e interactuar con el entorno. Como resultado, hay actos de discriminación dentro y fuera de este entorno que afectan drásticamente su calidad de vida. Por ello es necesario generar alternativas amigables para mejorar la interacción de estas personas con la sociedad en general.

Los talleres de producción audiovisual ponen en evidencia que este es el medio adecuado para generar interacción entre las personas con discapacidad auditiva y el entorno que los rodea. Al ser una herramienta útil para la manifestación y comprensión de las percepciones y pensamientos de las personas sordas, ofrece la oportunidad de comprender su forma de pensar y tener una mejor convivencia con ellas. 


\section{Bibliografía}

Anderson, I. F. (2016). Charles S. Peirce y el signo tres. Bold.

Tyler, I. (2018). Resitución de Erving Goffman: del poder del estigma al poder negro. The Sociological Review, 66 (4), 744-765.

ALVAREZ, G (2015) Discapacidad y sistemas alternativos de resolución de conflictos. Grupo Editorial Cinca, Madrid.

Cestero Mancera, A. M. (2006). La comunicación no verbal y el estudio de su incidencia en fenómenos discursivos como la ironía. ELUA. Estudios de Lingüística, N. 20 (2006); pp. 57-77.

Barragán Giraldo, D. F. (2016). Cartografía social pedagógica: entre teoría y metodología. Revista Colombiana de Educación, (70), 247-285.

Barrio, M. G., Fernández, M. R., \& García, S. Á. (2017). Metodología de producción para el desarrollo de contenidos audiovisuales y multimedia para MOOC. RIED. Revista Iberoamericana de Educación a Distancia, 20(1), 183-203.

Hoguín, J. (17 de febrero de 2017). Medio audiovisual como herramienta representativa del imaginario de las personas con discapacidad auditiva. (J. Fuentes, Entrevistador)

Producto audiovisual "Un mundo sin sonidos" [21 de marzo de 2017] Recuperado de: https://www.youtube.com/watch?v=9af0ss2hDl8 
